Helgoländer wiss. Meeresunters. 18, 169-193

\title{
Über Schalenabschliffe bei Cardium edule aus der Königsbucht bei List auf Sylt
}

\author{
R. Hollmann \\ Institut für Geologie und Paläontologie, Westfälische Wilhelms-Universität, Münster
}

\begin{abstract}
Shell abrasions in Cardium edule from the Königsbucht near List on Sylt. Two communities of the recent bivalve Cardium edule L. were collected in the tidal flats near List on the island of Sylt (Southern North Sea). The cockles were examined for abrasion marks on the surfaces of their valves arising from various causes. One important group of abrasions reveals traction marks restricted to the beak region, caused exclusively by valve movements. Another group is characterized by marks due to shell abrasions on the sea bed. The various types of abrasion marks have been studied in living cockles, both in the sea and in laboratory containers, and the results compared with those obtained on dead ones still connected by the ligament. In the sea, living Cardium edule were studied via Scuba-observations. Traction marks on the umbo support the conclusion that living cockles sometimes rest on their dorsal shell area. Holes in the convex umbo, umbonal facets, originate after death in situ or through shiftings to and fro on the sea bed, and also as a result of chemical or biological processes. The method applied and the criteria of shell abrasions employed are considered acceptable approaches to the interpretation of functional morphology and behaviour in fossil molluscs.
\end{abstract}

\section{EINLEITUNG}

Rezente Cardium edule L., deren Verbreitung beim Schwimmtauchen vor der Helgoländer Düne und in den Sandflächen nordöstlich außerhalb der OberkreideKlippenzüge beobachtet wurde, zeigen regelmäßige, mechanische Abnutzungsflächen an den harten, rein aragonitischen Klappen. Auf der Wirbelspitze und über dem dorsalen Buckel werden die charakteristischen Radialrippen dünn, zersplittern bei Beanspruchungen oder schleifen sich leicht $a b$. Vergleichbare Rippenschäden dehnen sich über die bauchig gewölbten Muschelflanken aus. Derartige Abschliffe treten sowohl an den nur vereinzelten lebenden als auch an den Schalen der verendeten Cardien auf, die häufig zu finden sind. Es erschien deshalb anziehend, die in die Schalen einschneidenden Zerstörungen morphologisch-topographisch $\mathrm{zu}$ sichten sowie ihren Ursachen nachzugehen. Auf Grund der vorliegenden Beobachtungen ist es durchaus möglich, bestimmte Verhaltensweisen postmortal allein an den Hartteilen zu erkennen und - durch Vergleiche - auch an fossilen Mollusken Aufschlüsse über besondere Lebensgewohnheiten zu erhalten.

Über Gehäuseschäden an lebenden Litorina litorea hat KEssel $(1937,1938)$ einige Erkenntnisse zusammengetragen. In die noch bewohnten Gehäuse dringen Cyanophy- 
ceen ein sowie auch der un Helgoland verbreitete Polychät Polydora ciliata, die das Schalen-Feingefüge lockern und postmortal zu einem artspezifisch verschieden schnellen Zerfall der Schalen führen. Schädigungen durch bohrende oder räuberische Organismen und ihre paläo-ökologische Bedeutung haben BoEKSCHOTEN (1966) und CARTER (1968) abgehandelt. Rekonstruktionen der Lebendstellung und Ökologie, die sich durch Epibionten auf fossilen Mollusken nachweisen lassen, führte SEILACHER (1954, 1960) durch. Die an bestimmte Schalenbereiche der Cardien gebundenen Abschliffe, die hier untersucht werden, hängen jedoch ursächlich mit deren aktiven Bewegungen in und auf den sandigen Meeresböden zusammen und deren durch Wasserströmungen und Sediment bestimmte Gesetzmäßigkeiten, in die sie passiv einbezogen werden.

Ausgehend von den Anregungen über die Erhaltung der Cardien vor Helgoland, werden Artvertreter aus dem Königshafen von Sylt betrachtet, die unter ökologisch stabilen Verhältnissen - und damit unter einfachen Versuchsbedingungen - aufgewachsen sind. Nahezu alle Gehäuse des umfangreichen, rezenten Materials zeigen, unabhängig vom Standort und von den individuellen Größen, morphologische Schäden, die zumeist auf beiden der zusammengehörenden Klappen spiegelbildlich-symmetrisch und regelmäßig wiederkehren, und zwar in gleicher Form, Ausdehnung und Intensität.

Eine Aufsammlung dünnschaliger, kleinwüchsiger und lebender Cardien stammt aus dem mehr oder weniger feinsandigen, an organischen Sinkstoffen reichen SchlickHochwatt der Tonnenlegerbucht mit ausgedehnten Ubermeereszeiten. Fehlende oder nur sporadische Sedimentverlagerungen führen in diesem Gebiet zur Bildung von Gyttja- und sapropelitischen Absätzen. Eine weitere Kollektion großwüchsiger, lebender oder auch verendeter Exemplare mit derben Schalen stammt vom Uthörner Außenwatt, aus einem feinsandigen Areal in Prielnähe mit kurzen Übermeereszeiten und aus einer nahegelegenen Strömungsrinne an der Grenze zur Mittleren Tide-NiedrigwasserLinie. Diese tiefliegenden Watten sind als das bisher reichste Cardium-Siedlungsgebiet bekanntgeworden (WoHLENBERg 1937, Abb. 1, p. 42).

\section{DER LEBENSRAUM VON CARDIUM EDULE}

Zur Deutung der typischen Bewegungs- und Verhaltensweisen der Herzmuschel Cardium und der dabei entstehenden, mechanischen Abschliffe sind im folgenden spezifische Lebensgewohnheiten des Tieres zusammengestellt. Neben eigenen Beobachtungen beim Schwimmtauchen und aus dem Watt wurden dazu lebende Cardien mehrere Wochen in einem Seewasserbecken gehalten. Bei zahlreichen Versuchen konnten die zu Gehäuse-Abschliffen führenden Bewegungen studiert und gegenüber dem Schriftum in wesentlichen Punkten ergänzt werden.

Cardium edule ist in der Nordsee an die feinsandigen Weichböden des Gezeitengürtels gebunden, ohne ausgesprochen korngrößen- und wassertiefenabhängig zu sein, und gehört in die Arenicola-marina-Gemeinschaft des Eulitorals. Damit reiht es sich in eine Gruppe von libero-hemisessilen Sandliegern und aktiv strudelnden SuspensionsFilterern der Infauna ein, die in einem noch jungen Flachmeer in den gegenwärtig aufgearbeiteten Pleistozänböden vorkommen. Gebiete mit starken Strömungen und Sedimentverdriftungen werden jedoch gemieden. 
Der Lebensraum ist durch den stetigen Wasserwechsel, durch erhebliche Temperatur- und Salzgehalts-Schwankungen, durch kurzfristig-episodische Wandlungen des Sedimentcharakters (Umlagerungen, Aufschüttungen) gekennzeichnet, mit denen sich die ökologischen Faktoren jeweils einschneidend ändern. Durch die extremen Forderungen an die Anpassung wird eine starke Einschränkung des Artenbestandes im Watt erzwungen, den der Individuenreichtum in einer Umgebung mit günstiger Sauerstoffund Nahrungsversorgung ausgleicht. Diesen ökologisch wechselhaften Bedingungen haben sich die Cardien angepaßt, deren biologische Aktivität speziell durch zeitweiliges Trockenfallen beschränkt und durch starke Temperatursprünge an der Oberfläche beeinträchtigt wird.

Aktive, weit- oder tiefreichende Wanderungen schließt die kugelige Gehäuseform der Cardien aus. Die kräftige Berippung dient zur Verankerung im Boden und erhöht gleichzeitig die Festigkeit der Schalen (BoETTGER 1932), auch zum Schutz der nur oberflächlich lebenden Tiere gegen Muschelräuber ( $z$. B. Möwen und Krebse). Die geringe Siedlungstiefe von 2 bis $4 \mathrm{~cm}$ wird durch die verkürzten Siphonen bestimmt, kann jedoch in $\mathrm{H}_{2} \mathrm{~S}$-reichen Böden aktiv vermindert werden.

Kurze Siphonen typisieren eine Reihe weiterer, opisthotruncater SandliegerMuscheln, die flachgründig siedeln und Plankton aus dem freien Wasser aufnehmen (vgl. SCHÄFER 1962, p. 180, Abb. 97).

Im Gegensatz zu den euthetischen Arten zeigt Cardium auch Eigenschaften des Pleurothetismus (ANTHONY 1905), die BOETTGer (1933) bei sonst in Weichböden vergraben lebenden Muscheln als Standortmodifikation auf festen Grundflächen erkannte, und zwar eine Verkürzung der Längsachse, insbesondere des Hinterendes. Die bauchiggerundeten Formen neigen zur Schalenverdickung, zu Wirbelabschliffen und Wachstumsanomalien. Asymmetrische Klappenformen entwickeln sich bei of umgelagerten Gehäusen jedoch nicht. Anscheinend ist bei Cardium edule die pleurothetische Gestalt konvergent durch Anpassung an den Lebensraum entstanden.

Verspülte Cardien reichen hoch über den Rand des Verbreitungsgebietes hinauf, bis in die Zone des unregelmäßig überfluteten und verfestigten Schlickwattes. Diese Abweicher vom ökologischen Optimum bleiben in verschiedenem Grade dünnschalig und kleinwüchsig (vgl. SwaN 1952). In den tiefliegenden Watten überwiegen dagegen Großformen, die auch in den stets submersen Bereichen siedeln. Die Untergrenze des Verbreitungsgebietes liegt $z$ wischen 6 bis $17 \mathrm{~m}$ Wassertiefe und wird durch den Heliotropismus der Cardien bedingt (SCHWARZ 1932, p. 453; VERWEY 1954, pp. 174, 178).

Freigespülte Cardien drehen sich in Wasserströmungen um die Längsachse der Gehäuse oder rollen über den Seeboden. Dabei werden die Klappen oft nicht völlig verschlossen, und die Siphonen schieben sich nur zum Teil, verkürzt, aus. Anscheinend werden die Tiere beim Rolltransport nur bedingt mechanisch beeinträchtigt. Mit einem kugeligen Gehäuse und einem beweglichen, kräftigen Fuß ausgestattet, ist es den Cardien möglich, auch noch in Gebieten mit beträchtlichen Sedimentverlagerungen zu überdauern. Dafür sind rasches Eingraben und schnelles Emporwühlen ausschlaggebend: Diese Beweglichkeit verlieren die Tiere während ihres ganzen Lebens nicht, und bereits nach 5 bis $10 \mathrm{~min}$ ist die Muschel in Lockersedimenten verschwunden.

Beim Trockenfallen wird das eingeschlossene Wasser in den Gehäusen teilweise durch Luft ersetzt. Uber die kräftigen Kiemen vermag Cardium auch begrenzt atmo- 
sphärische Luft aufzunehmen, die bei auflaufendem Wasser in kleinen Blasen abgegeben wird und ein knisterndes Geräusch erzeugt (THAMdrup 1935, p. 64).

In den beiden Siphonen, von denen sensorische Tentakel ausgehen, liegen empfindliche Blasenaugen. Im Versuch verhalten sich ausgewachsene Cardien ausgesprochen negativ phototaktisch und beginnen oder intensivieren ihre Grabbewegungen nach 2 bis 3 minütiger starker Bestrahlung. Die Tentakel sortieren ständig zu grobe, suspendierte Partikel aus dem Atmungs- und Nahrungs-Wasserstrom aus.

\section{DIE GRABBEWEGUNGEN VON CARDIUM EDULE}

Die mechanischen Eigenschatten des Ligamentes sind den ökologischen Anforderungen angepaßt: Oberflächlich siedelnde Muscheln, die oft zu aktiven Bewegungen gezwungen werden, besitzen ein festes, kräftiges Ligament (Hunter \& GRANT 1962, p. 378). Dagegen weisen ortsfeste und tiefbohrende Muscheln, wie z. B. Mya arenaria, im Alter nahezu unbewegliche Klappen auf: Der Zusammenhalt durch das Ligament wird weitgehend gelockert. Zumeist erfolgt die Klappenöffnung ungleichmäßig; das Hinterende, aus dem die Siphonen austreten, klafft weiter auf.

Bei den oberflächlich siedelnden Muscheln genügt zum Offnen der Klappen häufig allein die Spannung des kräftigen Ligamentes, dessen Elastizität durch den Wasserdruck innerhalb der Mantelhöhle unterstützt wird. Ansteigender hydrostatischer Druck innerhalb des Mantelhohlraumes, eines von den Siphonen isolierten, selbständigen Systems, läßt sich unmittelbar vor dem Auseinandertreten der Klappen beobachten. Dieser Druckanstieg ist bei Mya arenaria ausgeprägt, wobei nicht alle kurzfristigen Druckänderungen zu Klappenöffnungen führen (TRUEMAN 1954, p. 300 f.).

In stabiler Flankenposition auf dem Sediment liegende Cardien schieben ihren Fuß aus dem Mantelspalt suchend-tastend hervor, ohne daß sich die Klappen weiter öffnen. Dabei wird zuerst klumpig verschleimter Sand ausgestoßen, der beim plötzlichen Klappenschluß zwischen den Ventralrand und die Mantelsäume hineingeraten

Abb. 1: Kriechspuren von Cardium edule I. im Seewasser-Aquarium. Beim rudkhaften Vorwärtsstemmen der Muschel entstehen flache Furchen mit quergestreitten Seitenwällen. (Aufnahme: Dr. H. Hollmann)

Abb. 2: Cardium edule, Tonnenlegerbucht, lebend geborgen. Klappenlänge $=41 \mathrm{~mm}$ ! Muschelklappen in Verschluß-Stellung. Der markante Beruhrungskontakt beider Wirbelspitzen ist aufgehellt und als primäre Usur des 3. Modus stark abgeschliffen. $(2: 1)$

Abb. 3: Cardium edule, Tonnenlegerbucht, abgestorben und in Lebendstellung aus dem Boden gegraben. Ausbildung einer postmortalen Buckel-Facette in situ, einem durch mechanischen Abschliff und chemische Korrosion bedingten Einbruch in die dorsale Wölbung, die hier zusätzlich durch Algen-Bohrungen perforiert wird. $(3: 1)$

Abb. 4: Venerupis pullastra (MONTAGU), Helgoland-Nordreede, lebend geborgen. Bei der Verschluß-Stellung der Muschel werden die Wirbelspitzen gegeneinander gepreßt und bilden einen Schleifkontakt. $(2,5: 1)$

Abb. 5: Cardium edule, Tonnenlegerbucht, lebend geborgen. Abbrüche am ventralen Klappenrand des Winterringes 1963/1964, eine für diese Art seltene Wachstumsstörung. (2:1)

Abb. 6: Cardium edule, Tonnenlegerbucht, lebend geborgen. Ventrale Ausbrüche sowie SchalenUberdachung (unten) am Winterring 1964/1965. Folge der Winterringe vom Ventralrand: 1966/1967, mit dunklem Periostrakum; 1965/1966; 1964/1965, mit Wachstumsanomalien, denen proximal Störungsringe folgen. $(2,5: 1)$ 


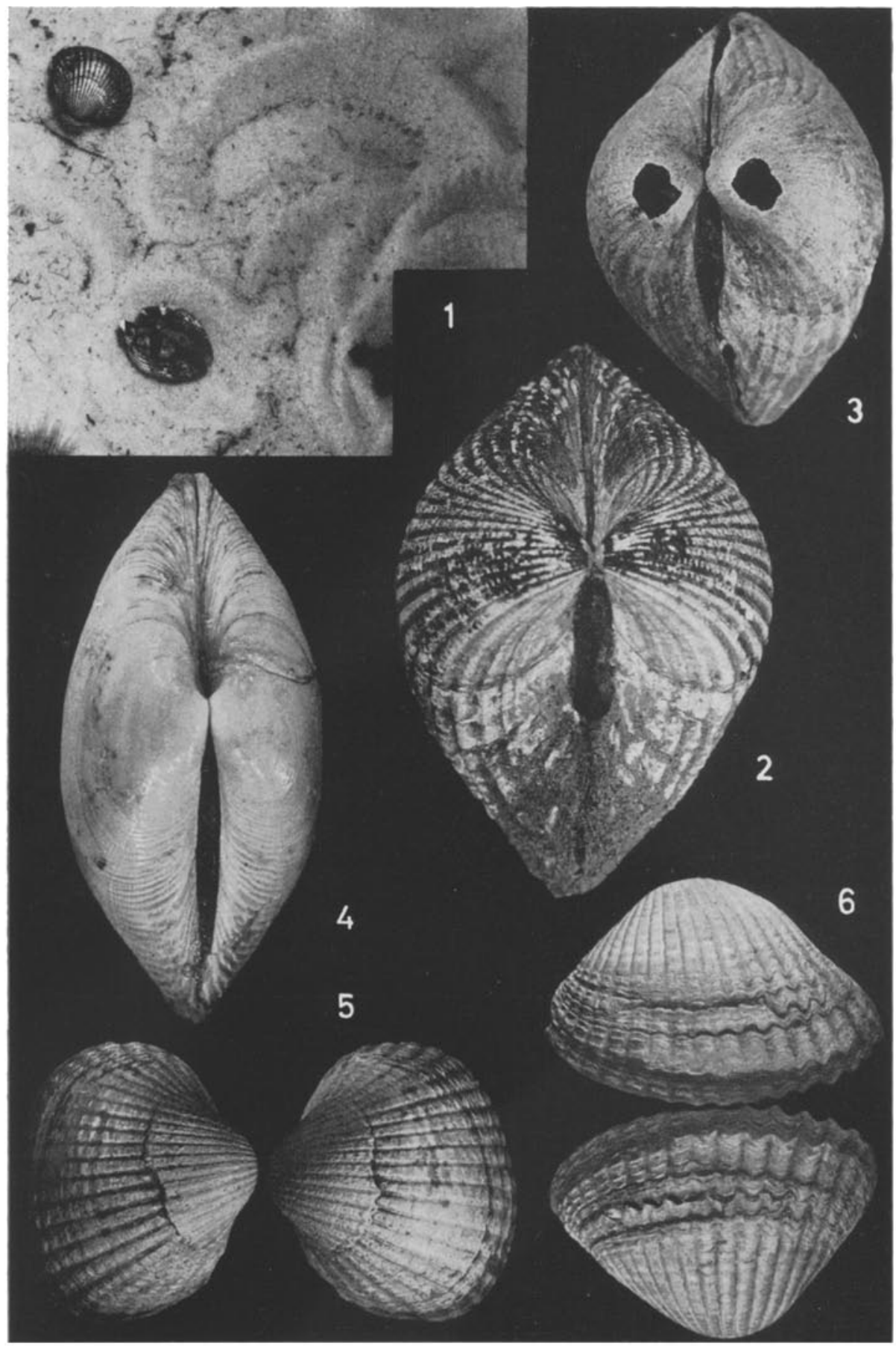


ist. Derartige plötzliche Klappenverschlüsse führen in den Watten zum bekannten "Spritzen" und sind Ausdruck einer Schreckreaktion.

Bei ausgewachsenen Cardien öffnen sich die Schalen hydraulisch durch das Vorschieben des Fußes, in den Körperflüssigkeit eingepreßt wird. Möglicherweise schwellen beim Offnen auch die ventralen Mantelsäume an und unterstützen den Druck der Klappen auf das umgebende Sediment. Der lang ausgestreckte, bewegliche Fuß wird hakenförmig nach unten abgewinkelt, bogig gekrümmt, ist nach allen Seiten frei beweglich und kann bis zum Wirbel hin umgeschlagen werden. Das Abbiegen des Fußes erfolgt oft am Klappen-Ventralrand im Bereich der Ringmuskulatur (SCHÄFER 1962, vgl. Abb. 112). Bohrend und wühlend stößt der keilförmige Fuß dann ins Sediment vor, wo er anschwillt und sich festklemmt.

Nach einer typischen Drehbewegung, die das Gehäuse aufrichtet, setzen dann beim Hinabklimmen in den Boden ruckweise Schüttel- und Schaukelbewegungen nach beiden Seiten ein. In gleicher Weise vermindert ruckhaft wiederholtes Offnen und Schließen der Klappen in rascher Folge den Widerstand des umgebenden Sedimentes und kann an teilweise eingegrabenen Tieren oft beobachtet werden. Dadurch werden thixotrope Sedimente verflüssigt oder das feste Sandkorn-Gerüst in mobilen Fließsand verwandelt. Anfangs reihen sich die Bewegungen in Abständen von 1/2 bis 1 min aneinander, folgen dann jedoch nach einer Phase intensiver Anstrengung in größeren Abständen aufeinander. Fast immer werden die Rüttelbewegungen durch einen kräftigen, vorwärtsgerichteten Spülwasser-Ausstoß aus der Mantelhöhle eingeleitet, der zu einem lokalen Anstieg des Wassergehaltes um das Gehäuse führt und auch dadurch das umgebende Substrat lockert. Ahnliche Verhaltensweisen sind von Mya arenaria und Venerupis pullastra (Trueman 1954) bekannt. Desgleichen beschleunigt der Rückstoß des gewaltsam aus den Siphonen gepreßten Wassers das Eindringen der Muscheln in den Boden.

Auf die das umgebende Sediment lockernden Schüttelbewegungen folgt die ruckhafte, fast sprungartige Gehäusedrehung, die durch eine plötzliche Kontraktion der Fußretraktoren ausgelöst wird. Dabei werden deren Insertionsbereiche am dorsalen Schalenrand zum Fuß hingezogen, und das Gehäuse dringt in den Boden ein, während sich die Schalenberippung parallel zur Bodenoberfläche orientiert. Der gewölbte Umriß und die Oberflächen-Skulptur ermöglichen es Cardium, sich rasch aufzurichten, ohne dabei seitlich abzugleiten.

Abb. 7: Cardium edule, Tonnenlegerbucht, lebend geborgen. Primäre, zu Lebzeiten gebildete Usur des 2. Modus, ein aufgehellter, rückweisender Schliffstreifen, auf dem die Radialrippen durch den Berührungskontakt der Klappen abgeschwächt sind. Wirbelspitze als primäre Usur des 3. Modus, glattgescheuert. (15:1)

Abb. 8: Cardium edule, Houlgate/Normandie, abgestorbenes Exemplar, Klappen noch ligamentär verbunden. Primäre Usur des 3. Modus an der Wirbelspitze. Durch einen schwach lädierten Querstreifen abgesetzt, folgt darüber die postmortal entstandene, sekundäre Usur

I. Form. Die Klappen schliffen sich gegenseitig bei einem Offnungswinkel von $40^{\circ} \mathrm{ab} .(10: 1)$

Abb. 9: Cardium edule, Tonnenlegerbucht, lebend geborgen. Primäre Usur des 3. Modus, eine stark beanspruchte, am Berihrungskontakt mit der Gegenklappe plangeschliffene Fläche an der eingekrümmten Wirbelspitze. Postmortale Einbrïche in diese, schon zu Lebzeiten geschwächte Abriebfläche führen zu Wirbelfacetten (PRATJE 1929). (13:1)

Abb. 10: Venerupis pullastra (MonTAGU), Helgoland-Nordreede, lebend geborgen. Primäre Usur des 3. Modus, die durch Klappenbewegungen des Tieres plangeschliffene Wirbelregion. $(13: 1)$ 

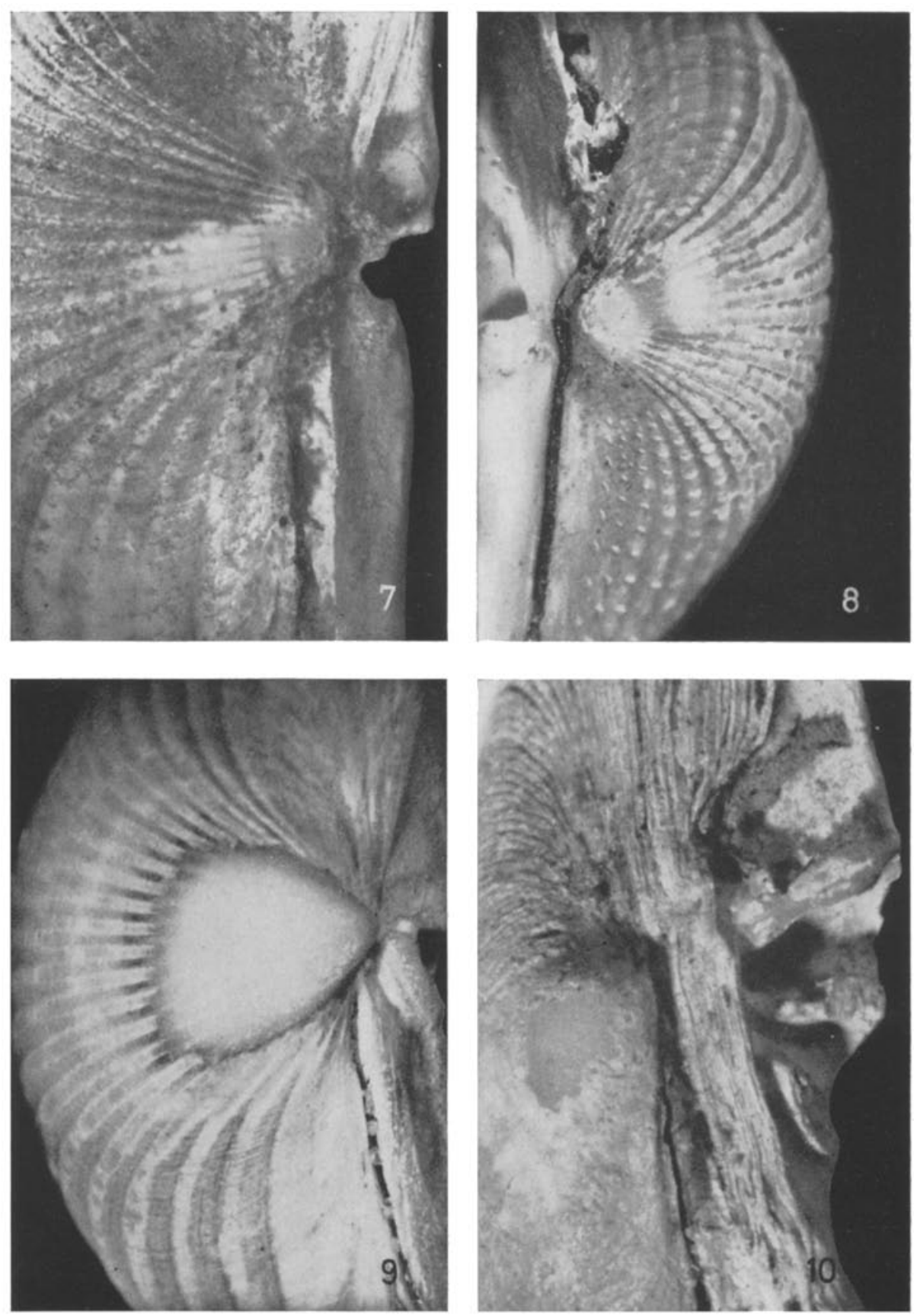
Das von Cardium bekannte Springen (bis $50 \mathrm{~cm}$ weit), das auf ein plötzliches Strecken des hakenförmig abgewinkelten Fußes zurückgeht, wurde nicht beobachtet. Es kann nur chemotaktisch durch spezifische Muschelräuber ausgelöst werden.

In den optimalen Siedlungsgebieten bildet Cardium flächenhaft verbreitet $\mathrm{Mu}$ schelpflaster; die Gehäuse liegen dicht gedrängt nebeneinander. Bisweilen entstehen dadurch den diagenetisch in klastischen Sedimenten geformten "Gerölleindrücken" vergleichbare „gezähnelte Mulden“, hervorgerufen durch die Ablösung des Mantelsaumes von der Schale (KREger 1940, p. 185, Abb. 18). Wachstums-Unregelmäßigkeiten bilden sich an den derben, widerstandsfähigen Schalen von Cardium selten aus. Im Gegensatz zu Mya arenaria (Schärer 1950) löst sich der Mantelrand erst unter extremen Umwelteinflüssen ab (Abb. 5 und 6).

Einzelne Individuen können in dichten Muschelsiedlungen auch durch ihre Nachbarn hochgedrückt werden oder stemmen sich aktiv zur Oberfläche empor, von wo sie durch Strömungen über weite Strecken und bisweilen auch in Massen verschleppt werden. Dichtbesetzte Kolonien können durch biologische (ZiegelmeIER 1963, p. 108) oder durch mechanisch-physikalische Faktoren, etwa durch Wasserströmungen (THAMdRuP 1935, Abb. 120; Schäfer 1962, Taf. 24, Fig. 54) oder Kälteperioden (WohlenberG 1937; Ziegelmeier 1964) dezimiert werden, obwohl Cardium kurzfristige Frostperioden unbeschadet übersteht (LINKE 1939, p. 276). Allerdings berichtet CARTER (1968, p. 33), daß gerade im Winter zusätzliche Verluste durch Möwen und andere Muschelräuber auftreten.

Ausgespülte Cardien auf festen Kleiböden (KREgER 1940, p. 175), in Prielen zusammengeschwemmte Tiere oder solche, die in Mytilus-Gespinste verstrickt sind, können auch auf der Sedimentoberfläche leben. Freiliegende Gehäuse verändern zuweilen durch peitschende Schläge mit dem Fuß und durch Wasserausstoß die Orientierung: Der weit ausgestreckte Fuß sucht dann züngelnd eine geeignete Stelle zum Eingraben. Diese Bewegungen erfolgen auf festen Substraten oft aus der stabilen Flankenlage oder in der stabilen Dorsalstellung, und das Gehäuse kann tagelang, auf den Flanken bzw. Buckeln liegend, gedreht oder fortgeschoben werden. Die Bewegungsphasen sind jeweils nur von kurzer Dauer. In dicht besiedelten Gebieten liegen Cardien häufig auch auf

Abb. 11: Cardium edule, Tonnenlegerbucht, lebend geborgen. Primäre Usur des 4. Modus, die narbige Buckel-Querstriemung. Der Schalenabrieb am Meeresboden ist durch aufgehellte, von den Zuwachslinien der Schale geführte Streifen nachzuweisen. $(13: 1)$

Abb. 12: Cardium edule, Uthörner Außenwatt, abgestorbenes Exemplar, Klappen noch ligament-verbunden. Uber der primären Usur des 3. Modus liegt der postmortale Abschliff. Die Schalen reiben sich bei passiven Klapp-Bewegungen aneinander und bilden dadurch einen hochgelegenen, rückweisenden Schliffstreifen aus. Dieser verbreitert sich bei seitwärts tordierenden Klappen nach außen und entspricht hier einem Offfnungswinkel von $70^{\circ}$. (13:1)

Abb. 13: Cardium edule, Tonnenlegerbucht, lebend geborgen. Primäre Usur des 5. Modus, der Buckel-Spiegel. Durch mechanische und chemische Einflüsse reibt sich die Skulptur flächig ab; die Abhängigkeit von den Anwachslinien ist deutlich. Die. Wirbelspitze wurde durch den KlappenSchleifkontakt stark usuriert. (13:1)

Abb. 14: Cardium edule, Uthörner Außenwatt, abgestorbenes Exemplar, Klappen noch ligament-verbunden. Sekundäre Usur IV. Form: Beim Schwoien und Treiben der gespreizten Klappen in stabiler Dorsalstellung auf dem Meeresgrund entsteht ein postmortaler Buckel-Spiegel. Oberste Lamellen platzen unregelmäßig aus der Schale heraus und führen zu Einbrüchen in die Dorsalwölbung, den Buckel-Facetten (vgl. Abb. 9). (13:1) 

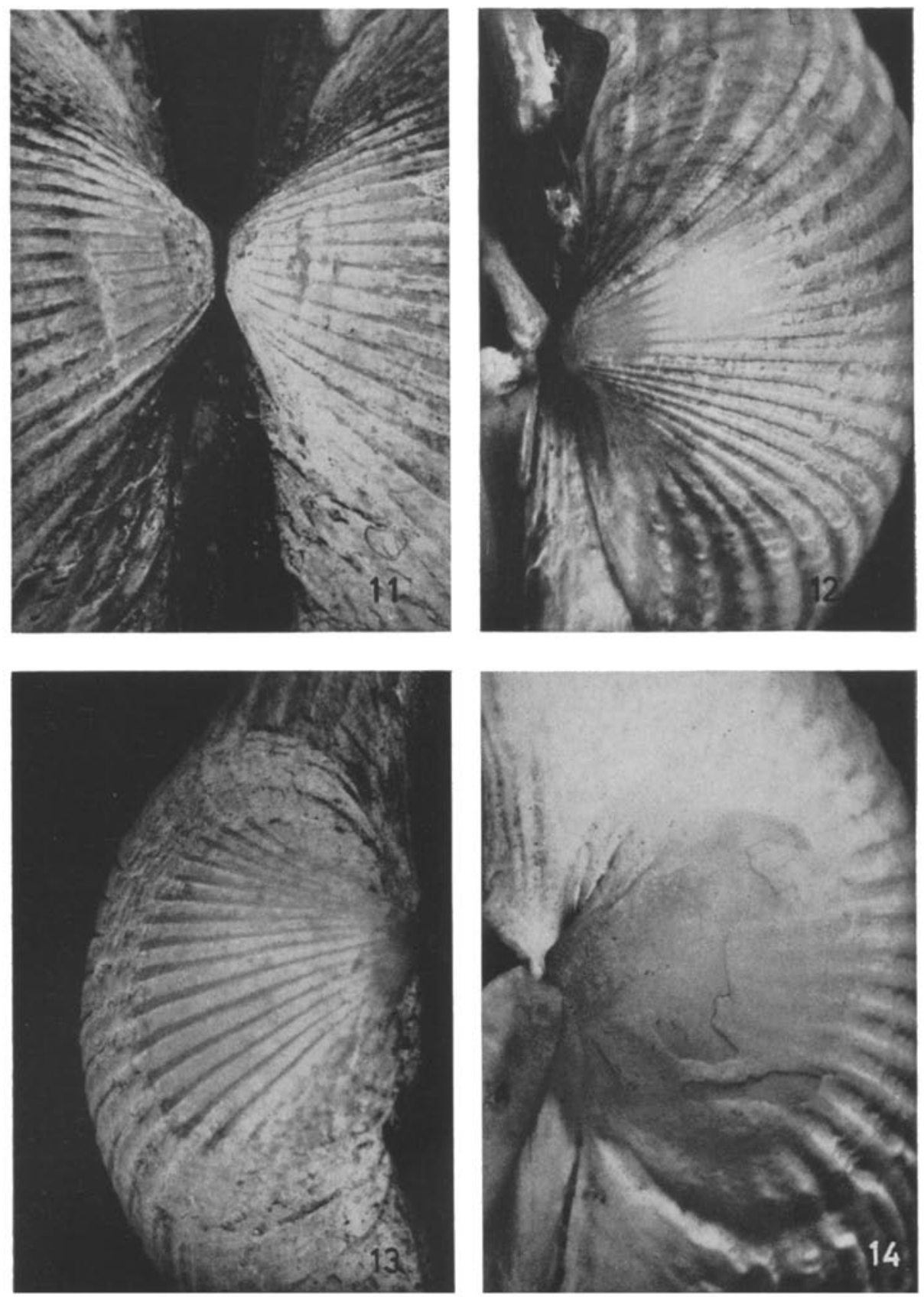
der Oberfläche und zeigen keinerlei Neigung, sich wieder einzugraben (HEcht \& MATERN 1930, p. 367; SCHUSTER 1951, p. 56).

Nur selten sind im Watt Muscheln zu beobachten, die sich über kurze Strecken auf der Ventralseite vorwärtsstemmen $(10$ bis $50 \mathrm{~cm}$ ), um ihren Standort zu verbessern. Dabei entstehen Furchen, Kriechspuren mit flachen Seitenwällen, die durch ruckweise-wippendes Vorwärtspflügen charakteristische Querstreifen erhalten (Abb. 1). Aktive, lange Wanderungen werden von adulten Tieren nicht unternommen: Cardium lebt ausgesprochen standort-gebunden (OrTON 1927, p. 261; Thamdrup 1935, p. 53, Abb. 120). Ahnliche, bereits strukturlose Seitenwälle umgeben teilweise eingegrabene Tiere, und über vollständig eingegrabenen Individuen wird der Boden zu einem flachen Hügel emporgedrückt.

Mytilus-Gespinste behindern zum Teil freigespülte Kolonien am erneuten Eingraben (Linke 1939, p. 277, Abb. 40; SchäfER 1962, Taf. 24, Fig. 54), und freiliegende Cardien werden durch Byssusfäden mit abgestorbenen Muscheln und anderem. Treibgut vernetzt. Zudem werden sie von Seepocken und Algen besiedelt, die den Atmungs- und Nahrungs-Wasserstrom mit ausnutzen, obwohl durch Epöken besiedelte Cardien ihre Siphonen viel weiter hinausstrecken und sich beim Trockenfallen auch ganz in den Boden zurückziehen können.

Abb. 15: Cardium edule, postmortale Besiedlung am ventralen Klappen-Innenrand durch thigmotaktisch orientierte, jugendliche Balanus balanoides L. (13:1)

Abb. 16: Cardium tuberculatum L., Houlgate/Normandie, abgestorbenes Exemplar, Klappen noch ligament-verbunden. Querschliff durch eine distale Radialrippe. Der Schalenbau wird bei $C$. tuberculatum und C. edule durch die Rippen-Furchen-Gliederung des Ostrakums bestimmt, desgleichen die Orientierung der radialstrahligen Aragonit-Fasern in den zahlreichen Lamellen. Im Bild sitzt ein erhabener Stachel auf dem Rippenkamm, dessen Feinbau dem der erhabenen Zuwachslamellen bei Cardium edule entspricht. Schäden an diesen empfindlichen Skulpturelementen zeigen den einsetzenden mechanischen Abrieb oder erste chemische Korrosionsschäden an. Das Periostrakum ist nur in den eingetieften Anwachslinien der Schale faltig und mit Anhängen erhalten, fehlt hier jedoch. Eine oberflächliche Deckschicht baut sich aus mehreren, schalen-parallelen Lamellen auf, dic teils isoliert sind und lateral auslaufen oder sich unnittelbar in die Lamellen des äußeren Ostrakums fortsetzen. Regelmäßig biegen die Lamellen des distalen, äußeren Ostrakums abwärts oder gehen in ein prismatisches oder auch fiederiggefächertes Fasergeflecht über. Die einzelnen Lamellen setzen sich in das Parallel-Gefüge des Ostrakums fort, das die proximale Schalentextur vom Wirbel bis zum ersten Winter- oder Störungsring allein bestimmt. Das Ostrakum besteht aus zahlreichen Lamellen von grober, kompakter, prismatischer Textur oder wird durch eine Füllmasse säuliger, fächeriger und radialstrahliger Aragonit-Fasern ersetzt. Eine dunkle Zone mit Pigment-Anhäufungen grenzt das Ostrakum nach unten hin ab. Sehr viel gleichmäßiger ist das Hypostrakum texturiert: Die typisch hellen und feinen Lamellen gleichen alle Aus- und Einbiegungen des Ostrakums mit zunehmendem Dickenwachstum aus. (25:1)

Abb. 17: Cardium tuberculatum L., Houlgate/Normandie, abgestorbenes Exemplar, Klappen noch ligament-verbunden. Querschliff durch den kalottenartig abgetrennten Dorsalbuckel, Radialrippen quergeschnitten. Das lamelläre Ostrakum (dunkel) sowie das Hypostrakum (hell) schließen sich den Ausführungen zu Abb. 17 an. Nach einer schwärzlich pigmentierten Einlagerung folgt innen weiteres, lamelliertes Hypostrakum. (13:1)

Abb. 18: Cardium tuberculatum L., Houlgate/Normandie, abgestorbenes Exemplar, Klappen noch ligament-verbunden. Längsschliff durch die Wirbelregion mit einer primären Usur des 3. Modus: Das Ostrakum (dunkel) dünnt zur Wirbelspitze hin keilförmig aus. Das Hypostrakum verliert gegen die Schliffläche an Dicke und lamellärer Vielfalt. Heterogene, von innen angelagerte Schalensubstanz zeichnet den Bereich des postmortalen Einbruchs an der Wirbelspitze vor. $(13: 1)$ 

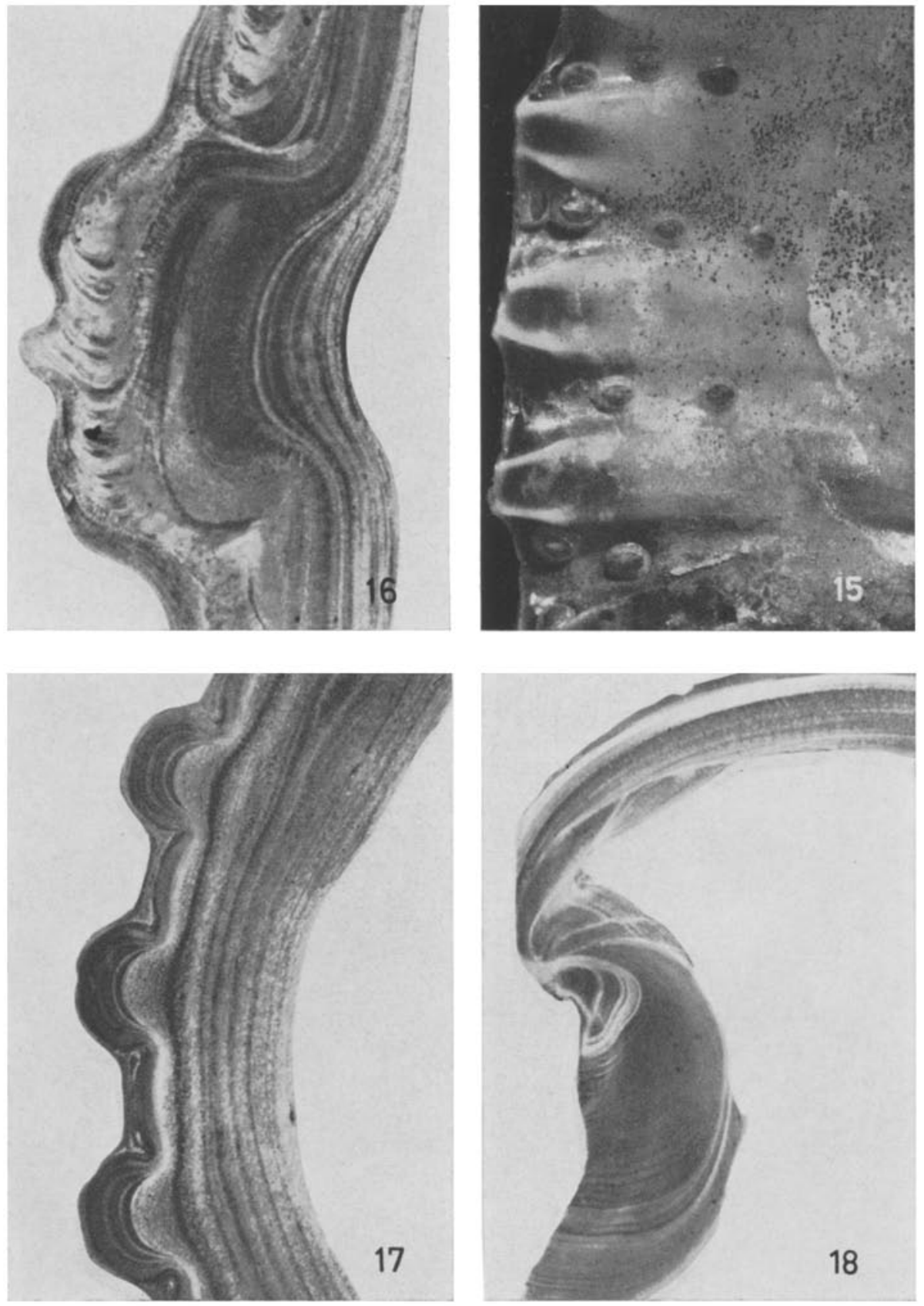
Cardien aus der Tonnenlegerbucht, an denen zahlreiche Byssus-Haftscheiben erkennbar sind, zeigen eine ausgesprochene Häufung von Störungsringen. Der Größe nach gehören die untersuchten Tiere zum Brutfall 1966 und haben den milden Winter 1966/67 überdauert. Entgegen früheren Beobachtungen können danach Cardien im Byssusgeflecht, die teilweise oder ganz freiliegen, mindestens bis zu zwei Jahren alt werden und sterben nicht vorzeitig ab. Die Angaben von KrEGER (1940, p. 680) sind dahingehend zu ergänzen.

\section{DIE WINTERRINGE VON CARDIUM EDULE}

Trotz der recht unterschiedlichen Gehäusegrößen und der verschiedenartigen, das Schalenwachstum fördernden oder hemmenden Biotope werden alle Schalen durch regelmäßig eingetiefte Wachstumsunterbrechungen überprägt. Als spezifisches Kennzeichen kehren sie auf den zusammengehörenden Muschelklappen konstant und in festem Rhythmus wieder. Sie liefern Anhaltspunkte über das ontogenetische Alter der betreffenden Tiere (ORTON 1927; Vogel 1959) sowie über ausgeprägte Veränderungen der Umwelteinflüsse während des Wachstums. Die Deutung der Winterringe wird durch die über mehrere Jahre ausgedehnten Beobachtungen KREGERs (1940) gesichert. Für die Hochwatten der Tonnenlegerbucht müssen auch andersartige, ökologisch bedingte Einschnitte in den Lebensablauf berücksichtigt werden. Trockenfallen, dadurch bedingter Nahrungsmangel, Temperatursprünge, plötzliches Uberschütten oder Freispülen verursachen Störungsringe allgemeiner Art, die sich bei umfangreichem Material teils auf Grund ihrer geringen Eintiefung erkennen und von Winterringen unterscheiden lassen. Als nicht zeitgebundene Bildungen folgen die Störungsringe arhythmisch aufeinander.

Nach einer Orientierung an den markanten Wachstums-Unterbrechungen 1962/ 1963 und 1965/1966 wird die Auszählung der Winterringe vom ventralen Schalenrand her vorgenommen. Hier drängen sich die Einschnitte in den Schalenzuwachs bei nachlassender Wachstums-Intensität mit steigendem Alter, treten durch schuppig verfälteltes Periostrakum deutlich hervor und verlieren in milden Wintern ihre charakteristische Ausbildung. Die aufgeführten Stillstandsperioden im Schalenzuwachs treffen alle Cardien der verschiedenen Jahresklassen mit gleicher Stärke und in gleichem Rhythmus. Eine Ausnahme bilden Cardien aus Prielen, deren wechselvolles Schicksal sich in zahlreichen, z. T. gravierenden Störungsringen widerspiegelt. Ihre Zuordnung zu den einzelnen Jahresgruppen erfolgte nach eingehenden Vergleichen nur mit Vorbehalt.

Winterring 1966/1967. An den im Juli 1967 ausgegrabenen Cardien liegt am ventralen Außenrand eine schwache Wachstumsunterbrechung, die einem milden Winter zuzuordnen ist. Bei den älteren Cardien ist der Zuwachs im Sommer 1966 auffallend gering, möglicherweise als Folge der verhältnismäßig spät im Jahr einsetzenden Schalenbildung bei niedrigen Wassertemperaturen und Plankton-Armut nach einem kalten Winter. Bei diesen älteren Tieren fällt der Winterring 1966/1967 häufig ganz aus und wird durch einen Streifen mit mehreren schwachen Störungsringen ersetzt (vgl. ORTON 1927, p. 261).

Winterring $1965 / 1966$. Dorsalwärts folgt ein tiefer Einschnitt in die 
Schalen, der durch schuppig-lamelliert wucherndes, dunkelbraunes Periostrakum als prägnante Unterbrechung an allen Muschelklappen hervorsticht.

In diesem Winter entstanden Wachstums-Anomalien, die sonst bei Cardium selten sind: Bisweilen löste sich der Mantelsaum vom vorderen Klappenrand ab, möglicherweise beim Eingraben in leicht gefrorene Böden oder durch unmittelbare Frosteinwirkung, so daß der Schalennachwuchs treppig abgesetzt ist.

Winterringe $1964 / 1965$ und $1963 / 1964$. Die beiden nächstälteren Winterringe sind nur mäßig eingetieft und verfärbt. Gelegentlich lassen sie sich nicht mehr eindeutig gegen die Störungsringe allgemeiner Art abgrenzen.

Wint e r r ing $1962 / 1963$. Wiederum zeichnet sich eine tiefe, typische Einkerbung in die Schalen mit dichtem, dunklem Periostrakum ab, die Gravur eines strengen Winters (ZIEGELMEIER 1964).

Winte $\mathrm{r}$ ring $1961 / 1962$. Diese Wachstums-Unterbrechung zieht sich als schmale, scharf eingeschnittene Linie um den dorsalen Buckel und stellt bei den meisten Cardien die erste Markierung dar.

Winterring 1960/1961. In einzelnen Ausnahmen schneidet eine noch ältere, scharf eingravierte Wachstumsunterbrechung den Wirbel kalottenartig ab. Diese im untersuchten Material seltenen Stücke dürften aus dem Brutfall 1960 stammen.

Cardien mit einem Winterring stammen aus dem Brutfall des vorhergegangenen Sommers und stehen im 2. Lebensjahr: Demnach sind die seltenen Exemplare mit 7 Winterringen achtjährig.

\section{ANALYSE DER ZU LEBZEITEN ENTSTEHENDEN SCHALENABSCHLIFFE BEI CARDIUM EDULE}

Trotz des zahlenmäßig umfangreichen Materials aus der Tonnenlegerbucht (Abb. 19) hält sich die Variabilität der Cardium-Langachsen zwischen 20 bis $30 \mathrm{~mm}$, der Durchschnitt (50 Exemplare) liegt bei $24 \mathrm{~mm}$. Einzelne, verschwemmte Großformen erreichen Längen von 40 bis $42 \mathrm{~mm}$.

Die Längen der Cardien aus dem Uthörner Außenwatt (Abb. 20) variieren zwischen $13 \mathrm{~mm}$ (Jahrgang 1966!), sonst zwischen 17 bis $43 \mathrm{~mm}$ mit einem Durchschnittswert (50 Exemplare) von $36 \mathrm{~mm}$. Davon sind rund $25 \%$ kleinwüchsige, verdriftete Tiere der Hochwatten.

Bohrende Algen (BoEkschoten 1966) machen die Schalen brüchig und porös; speziell die Jahresklassen 1962 und 1963 wurden stark befallen. Cardien aus dem Sommer 1961 sind unterschiedlich, jedenfalls stets schwächer angebohrt. Dies ist möglicherweise ökologisch bedingt; rom Sommer 1964 an bleiben die nadelstich-artigen Bohrungen aus.

(a) Flankenlage a uf Weichböden. Gewöhnlich behalten lebende Cardien diese Gehäuse-Orientierung nur kurzfristig bei. Sie hinterläßt keine kennzeichnenden, spezifisch nur dadurch verursachten Abriebspuren an den Schalen, obwohl freigespülte Tiere oft auf einer der Flanken liegen und alle Fortbewegungen mit Drehungen aus dieser Position eingeleitet werden.

(b) Eindringen mit dem Vorderende durch schaukelnd-ruckhaftes 
Klimmkriechen sowie ausnahmsweise aktive Ortsveränderungen durch rüttelnd-wippendes Vorwärtspflügen (Stemmkriechen!) auf Weichböden. Auch dabei entstehen keine typischen Abschwächungen der Skulptur; die Berippung ist vorne und hinten in gleicher Art erhalten, insbesondere auf dem Dorsalbuckel.

(c) P a s sive s Verdrifte n ausgespülter Cardien durch Rolltransport über den Boden prägt sich nicht durch kennzeichnende Schalenabschliffe aus. Sie sollten gegebenenfalls allseitig in gleicher Stärke auftreten.

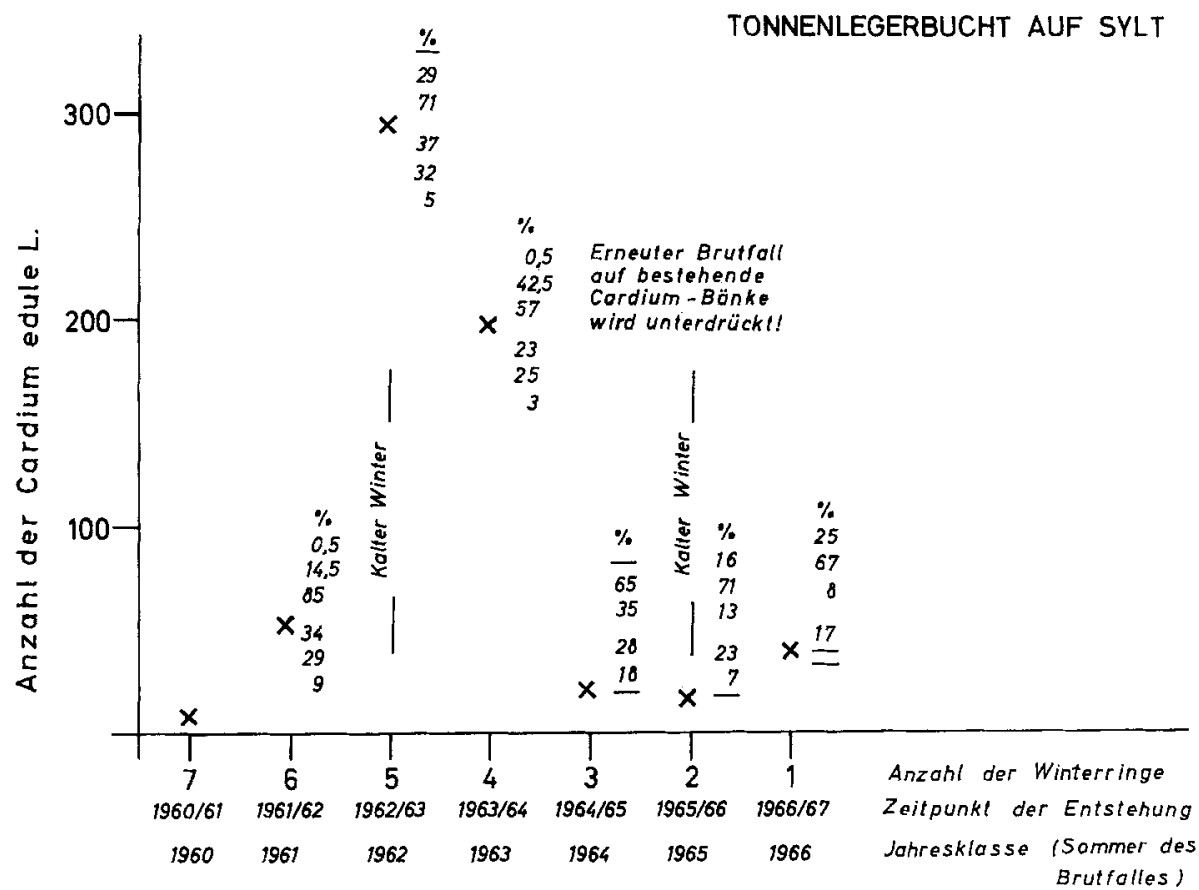

Abb. 19: Jahresklassen $(X)$ und Arten des Schalenabschliffes (\%) bei Cardium edule aus der Tonnenlegerbucht (im Juli 1967 lebend geborgen)

Allerdings sind flächige Rippenabschwächungen auf den gewölbten Flanken beider Klappen in typischer Form ausgebildet ud gehen auf die vorstehend genannten Ursachen zurück. Dabei werden das Periostrakum und die Berippung im Mittelfeld der Schale abgeschürft: Besonders empfindliche Skulpturelemente stellen die feinen, erhabenen Zuwachslamellen auf den Rippenkämmen dar, die erkennen lassen, daß die Cardien unterschiedlich lange und häufig auf einer der beiden, verschieden stark $a b$ geriebenen Seiten gelegen haben. Erste schwache Abschliffe an den Rippenkämmen der Flanken stellen sich bei Cardien im 2. Jahrgang ein, sind jedoch niemals so gleichförmig und kräftig wie die dorsalen Abschliffe. Mit zunehmendem Größenwuchs verschiebt sich die laterale, bodenberührende Fläche ventralwärts auf immer jüngere Schalenpartien. Abgeriebene und stark aufgehellte Flächen von gerundetem Umriß liegen bei kleinwuichsigen Tieren genau auf der Klappenmitte, sind bei großen Exemplaren un- 
mittelbar hinter die Mitte verlagert und rücken nur bei $5 \%$ der untersuchten Schalen vor die Mitte. Darin dürften unterschiedliche Schwerpunktslagen der Tiere ihren Ausdruck finden.

(d) Spiegelbildlich-symmetrische Läsionen der Berippu n beider Klappen durch Abrieb, die nahezu an allen Cardien auftreten, bilden sich am Berührungskontakt der Wirbelspitzen beim Offnen und Schließen der Gehäuse. Sie korrespondieren mit einem Klappen-Ơffnungswinkel von 10 bis $15^{\circ}$ (Abb. 2).

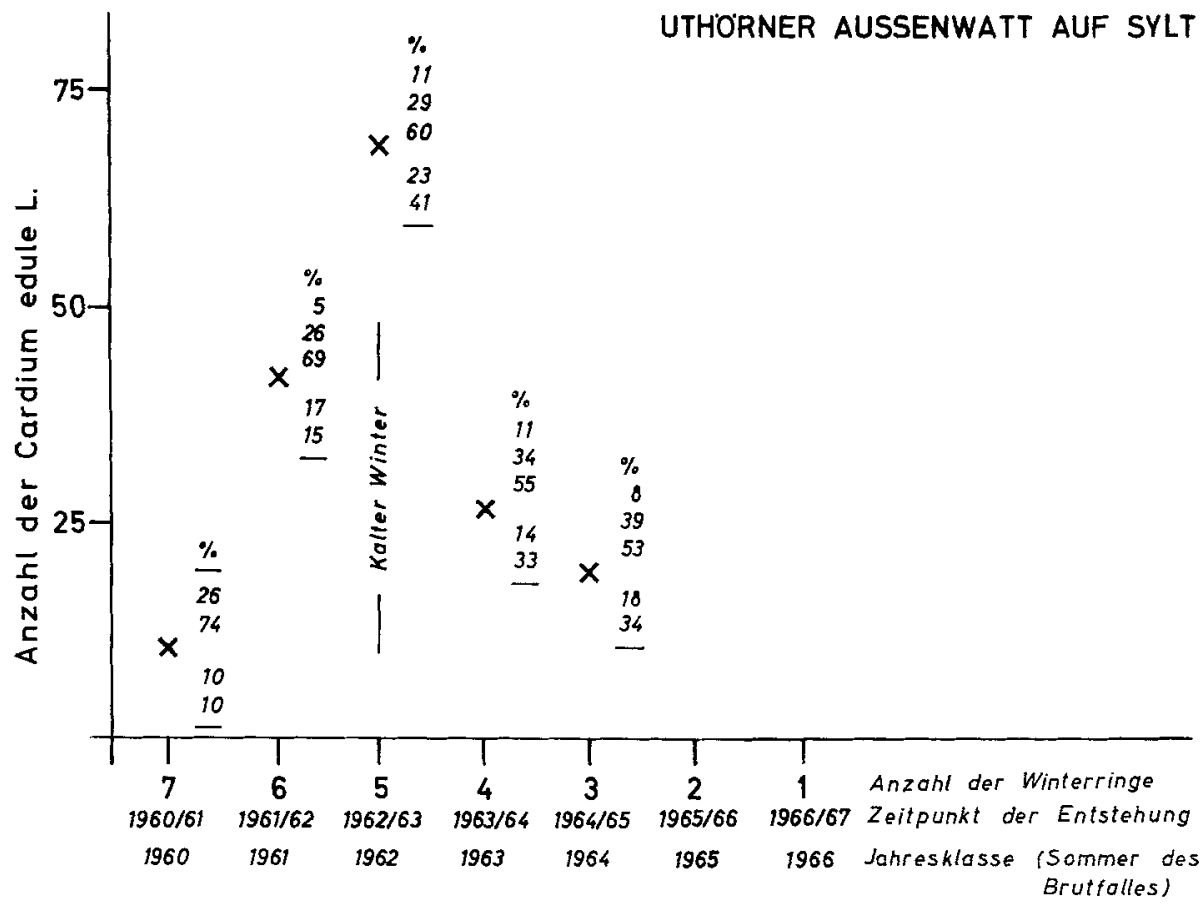

Abb. 20: Jahresklassen (X) und Arten des Schalenabschliffes ( $\%$ ) bei Cardium edule vom Uthörner Außenwatt (in September 1967 lebend geborgen)

Mit der Länge von 1,5 mm erreichen Cardien ihre adulte Gestalt und sind, bis auf die glatte Wirbelspitze, die der überdauernde Prodissoconch einnimmt, berippt (Creer 1960, p. 255). Die feine, erste Berippung um den unskulptierten Prodissoconch geht aber in der Folgezeit verloren.

Beim Eingraben in den Boden und bei allen Bewegungen der flachgründig-ortsgebunden siedelnden Tiere werden die Klappen unzählige Male geöffnet und verschlossen. Dieses instinktive Verhaltensmuster führt zu prägnanten Schalenschäden: Die aufeinandertreffenden, eingekrümmten Wirbelspitzen reiben sich aneinander in verschieden starkem Maße ab. 
render Streifen oder ein gelängtes Oval mit nach hinten weisender Längsachse abgeschliffen wird. Auch noch postmortal bestimmt diese im Bauplan begründete Asymmetrie die Form und Lage der Schliffschäden (p. 172).

Feines, zwischen die Wirbelspitzen eindringendes Sediment begünstigt dabei sicherlich den Schalenabrieb. Gerade die stark abgenutzten Flächen (primäre Usuren des 2. und 3. Modus) werden oftmals von einem Schlick-Randwall umgeben.

Im übrigen ist die flächige Ausdehnung der Abschliffe am Berührungskontakt beider Klappen der Gehäusegröße proportional. In Gebieten mit häufiger Aufarbeitung werden die Schliffe ebenfalls relativ groß und sind zusätzlich mit Skulpturabschürfungen auf den Flanken und dem dorsalen Buckel verbunden. Zusammengenommen können sie als eindeutiges Kriterium für instabile Biotope mit Sedimentverlagerungen gelten.

Diese zu Lebzeiten entstandenen Schalenabnutzungen nennen wir primäre Usuren im Gegensatz zu gelegentlich ähnlich geformten Schalenschäden, den sekundären Usuren, die ausschließlich postmortal durch passive Klapp-Bewegungen bereits verendeter, aber noch schloß- und/oder ligament-verbundener Muschelklappen in den Grundströmungen am Seeboden entstehen. Sowohl die primär als auch die sekundär usurierten Flächen gehen durch Zwischenformen ineinander über und werden oft von Flankenabschliffen begleitet.

Sekundäre Usuren, als typisch postmortale Veränderungen, treten an den lebend geborgenen Cardien der Tonnenlegerbucht und des Uthörner Außenwattes nicht auf!

\section{Morphologie und Funktion der primären Usuren}

Trotz der Variabilität der Schlifformen an den Gehäusen korrespondieren sie auf zusammengehörenden Klappen spiegelbildlich-symmetrisch miteinander. Entsprechend eng begrenzt ist jeweils der Spielraum ihrer Entstehungsmöglichkeiten. Die Erhaltung der Feinskulptur auf der Wirbelspitze oder auf den dorsalen Buckeln ist von der Größe, vom Alter, von der Widerstandsfähigkeit der Berippung sowie vom Biotop abhängig. Sonst ist die Schale jedoch, in Anpassung an den Lebensraum, Abschliffen und Einbrüchen gegenüber relativ widerstandsfähig (vgl. auch Abb. 19 bis 21).

\section{Die Skulptur der Wirbelspitzen}

1. Modus: Intakte Wirbelspitzen, Periostrakum und Rippen vollständig erhalten, bisweilen mit mineralischen Uberkrustungen sowie erste Andeutungen eines Schleifkontaktes der Wirbelspitzen.

Unter den Exemplaren aus der Tonnenlegerbucht fällt der Anteil von $25 \%$ im 1. Jahrgang auf $16 \%$ im 2. Jahrgang und fehlt in den älteren Jahresklassen ( 0 bis $0,5 \%$ ). Im weniger umfangreichen Material vom Uthörner Außenwatt hält sich der Anteil um $10 \%$, sinkt dann jedoch plötzlich ab und entspricht damit den Angaben in Abbildung 21.

2. Modus: Aufgehellte oder mattglänzende, \pm starke Schliffstreifen, auf denen 
die Radialrippung der Wirbelspitzen abgewetzt ist, oder leichte, längliche Abriebflächen ohne Skulptur. Periostrakum, Rippen oder mineralische Anflïge fehlen. Die nach rückwärts gerichteten, weißen Schliffstreifen verbreitern sich zunehmend und tendieren $z u$ abgerundet-ovalen Formen (Abb. 7).

Mit zunehmendem Alter verringert sich dieser Modus des Schalenabschliffes in den ersten drei Jahresklassen aus der Tonnenlegerbucht nur geringfügig (von 71 bis $65 \%$ ), fällt dann jedoch gleichmäßig stark ab und erreicht im 6. Jahrgang den Wert von $15 \%$.

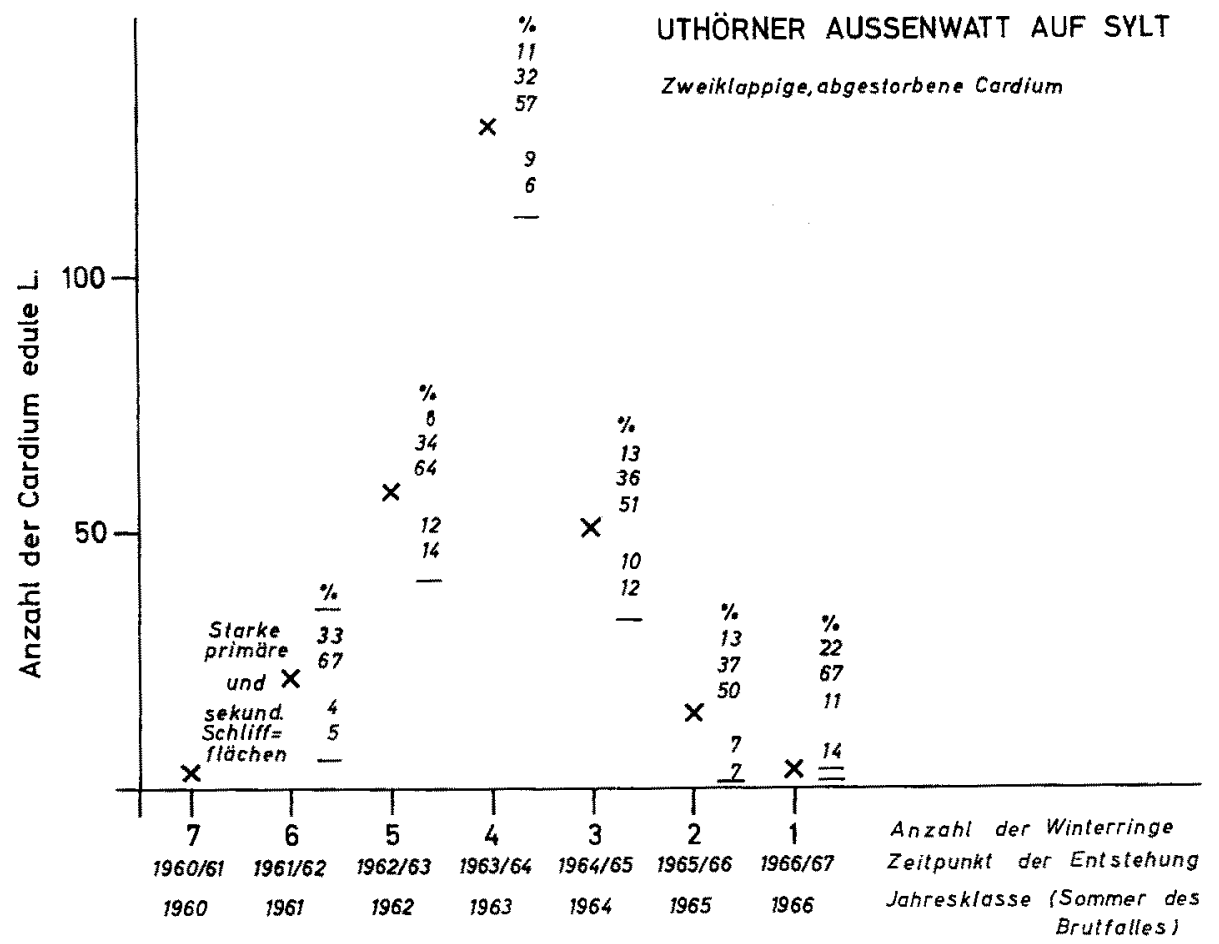

Abb. 21: Jahresklassen $(X)$ und Arten des Schalenabschliffes (\%) bei Cardium edule vom Uthörner Außenwatt (bis zum September 1967 abgestorben, aber noch zweiklappig durch das Ligament verbunden)

Im Uthörner Außenwatt liegt der Anteil der Schalen mit einem Schliffstreifen dagegen bereits anfänglich unter den vergleichbaren Werten aus der Tonnenlegerbucht, zeigt jedoch auch hier eine mit zunehmendem Alter fallende Tendenz. Diese ist, ebenso wie in Abbildung 21, schwächer ausgeprägt. Die ersten beiden Jahresklassen fehlen in den Aufsammlungen vom Uthörner Außenwatt. Bei großwüchsigen, alten Cardien wird der Schliffstreifen breit und kräftig ausgebildet.

3. Modus: Breite, geglättete oder blätterig-schuppige, stark beanspruchte $\mathrm{Ab}$ riebflächen, überwiegend von gelängt-ovaler, abgerundeter Form. Dabei wechselt die Dicke der abgetragenen Schalenschicht beträchtlich, und zwischen nur oberflächlichen Abnutzungen, ebenen, uneben-welligen oder gar konkaven Abschliffen an den Wirbelspitzen finden sich zahlreiche Ubergangsformen (Abb. 9). 
Mit ansteigendem Alter fallen die Cardien aus den beiden vorstehenden Gruppen diesem Modus zu, von einem geringen Anteil von 8 bis $13 \%$ im 1. und 2. Jahrgang bis auf $85 \%$ im 6. Jahrgang. Die Zunahme erfolgt in der Tonnenlegerbucht nahezu linear. Die stark beanspruchten Abriebflächen werden im 1. Lebensjahr zum größten Teil vollständig ausgebildet, bei den kleinwüchsigen Cardien als runde, kleine Schliffflächen. Im Uthörner Außenwatt (Abb. 20 und 21) werden die Wirbelspitzen im wesentlichen bis zum 2. Jahrgang stark abgeschliffen; ihr Anteil erhöht sich dann mäßig von 50 auf $75 \%$.

Anscheinend geben die empfindlichen Rippen der kleinwüchsigen Cardien aus der Tonnenlegerbucht, die zudem weiteren destruktiven Einflïssen ausgesetzt sind, den mechanischen Abrieb genauer wieder als die großwüchsig-derbberippten des Uthörner Außenwatts. Bei den ausgedehnten Übermeereszeiten im Hochwatt dürften die Klappen häufiger bewegt und intensiver abgeschliffen werden. Die genannten Divergenzen in den Schalenschäden des 2. und 3. Modus in den Jahresklassen 1961 bis 1964 rom Uthörner Außenwatt könnten dadurch erklärt werden.

\section{Die Skulptur der Buckel}

Zu Lebzeiten entstehende Abschliffe am dorsalen Buckel treten bei vielen Muscheln auf. Bei ausgewachsenen Cardien werden die zart skulptierten Teile der Klappen am Buckel bei allen Bewegungen im Sediment weit häufiger als die jüngeren, distalen Schalenpartien abgerieben. Zudem werden die ältesten Teile der Klappen von den standort-gebundenen, festliegenden Cardien, soweit sie aus dem Boden herausragen, vom im Wasser treibenden Feinsand abgeschliffen. Derartige mechanische Schädigungen sind nur schwer von den chemischen Zerstörungen der Berippung zu unterscheiden. Durch Verwitterungseinflüsse, insbesondere im Hochwatt der Tonnenlegerbucht, werden das ohnehin dünne Periostrakum und das Konchiolin-Gerüst der Schale angegriffen, so daß die ältesten Schalenpartien zuerst in ein leicht lösliches, poröses Maschenwerk aufblättern. In den Gyttja- und sapropelitischen Böden der Tonnenlegerbucht ist die chemische Lösung von der $\mathrm{H}_{2} \mathrm{~S}$-Konzentration des Bodenwassers abhängig; im Uthörner Außenwatt dürfte der $\mathrm{CO}_{2}$-Gehalt eine entsprechende Rolle spielen (MosEBACH 1952). Biologischer Abbau der empfindlichen Skulptur auf den Cardium-Buckeln ist außerdem in der Tonnenlegerbucht auf nadelstichartige Algenbohrungen zurückzuführen.

Auf festen Kleiböden, ebenso aber auch auf feinklastischen Weichböden, nehmen die Cardien zwei stabile Lebendstellungen ein, die zu Abschliffen führen.

In der stabilen Flankenlage werden unzählbare Dreh- und Stemmbewegungen vollführt, ohne daß das Tier in den Boden eindringt (p. 176). Außerdem ist auch zu Lebzeiten eine stabile Dorsalstellung möglich, bei der sich die Cardien in Umkehrung zur Lebendstellung hochkant aufrichten.

Mechanisch bietet das abgerundete Gehäuse mit dem breiten Buckel eine sichere Standfläche, die nach den vorliegenden Beobachtungen gelegentlich auch ausgenïtzt wird. Der Einströrnungs-Sipho gelangt durch diese Orientierung in höhere Wasserschichten, in denen die Versorgung mit sauerstoff- und nahrungsreichem Wasser günstig ist. Demgegenüber bleibt der dem abführenden Wasserstrom schubweise beigemengte 
Kot gravitativ am Boden. Solche Umkehrung bringt, insbesondere in Stillwasserbereichen, merkliche Vorteile für den Stoffwechsel mit sich. Allerdings ist Cardium dorsal-ventral orientiert, und die Umkehrung stellt den Ausnahmefall dar!

Bei den in der Brandungszone "angelnden" Muscheln beobachtete SeILACHer (1953), daß sie den Einströmungs-Sipho gegen den nahrungbringenden Rückstrom der Wellen richten und in der über den Boden gleitenden Fracht den Detritus aufnehmen. Aus den gleichen Gründen wenden Cardium und Mya arenaria auffallend häufig in 3 bis $6 \mathrm{~m}$ Wassertiefe im gezeitenlosen Südbecken von Wilhelmshaven ihre Ventralseite dem Ufer zu. Damit liegen die Siphonen in den Fallinien der submersen Böschungen hintereinander und fangen hangabwärts verlaufende Wasserströmungen auf.

4. Modu s: Schmale, streifige Buckel-Querstriemung, in deren Bereich die Berippung abgeschwächt ist oder völlig fehlt und die - ausgeprägt - als weißes Band quer über den Buckel verläuft (Abb. 11).

Diese hellen Querstreifen entsprechen diskontinuierlichen, symmetrischen Läsionen an beiden Buckeln. Sie entstehen durch das Zusammenwirken der vorgenannten Einflüsse und setzen nach Dünnschliff-Befunden an Störungs- oder Winterringen im frühen Jugendstadium an, die durch mechanische und chemische Schädigungen sichtbar hervortreten. Durch den raschen Schalenzuwachs im 1. bis 3. Lebensjahr werden sie als auseinandergezogene Narben deutlich.

5. Modus : Flächiger Abschliff über dem gesamten Dorsalbuckel als weißer Buckel-Spiegel, Ausdruck starken Schalenabriebs. Skulptierung abgeschwächt oder völlig fehlend. Flächen mattiert, schuppig oder glatt poliert. Der Buckelspiegel wird gelegentlich durch einen schmalen Querstreifen erhalten gebliebener Rippenreste von den Schlifflächen der Wirbelspitze abgetrennt (Abb. 13).

Mechanische, chemische und biologische Ursachen zerstören die Berippung beider Buckel gleichmäßig-kalottenartig. Mit zunehmendem Größenwuchs dehnen sich auch die Ab̈schliffe weiter aus. Die Formen des dorsalen Buckelabschliffes sind häufig mit den fortgeschrittenen Stadien des Wirbelabriebs (2. und 3. Modus) gekoppelt. Im Uthörner Außenwatt kommen in den Jahresklassen 1962 bis 1964 Buckelspiegel überdurchschnittlich häufig vor (Abb. 20) und dürften vorwiegend auf Sandschliff zurückzuführen sein.

Der Anteil der Buckel-Abschliffe unterliegt an den ausgezählten Exemplaren erheblichen Schwankungen und geht auf Schwächestellen im Schalenbauplan, ebenso aber auch auf ökologische, an den Standort gebundene Wechselwirkungen zwischen den verschiedenen, destruktiven Einflüssen zurück. Eine mit zunehmendem Alter ansteigende Tendenz des 4. und 5. Modus ist an den Cardien aus der Tonnenlegerbucht zu beobachten; die Durchschnittswerte des gesamten Materials für diese Modi liegen zwischen 10 und $20 \%$.

6. Modus: Postmortal gebildete Buckel-Facetten, flächige, eckig begrenzte Durchbrüche der Dorsalwölbungen treten bei noch in Lebendstellung im Boden befindlichen, aber bereits schlick-erfüllten Exemplaren aus der Tonnenlegerbucht auf. Ih: Anteil bleibt gering, tritt erstmals im 5. Jahrgang in Erscheinung und steigt bezeich" nenderweise in den folgenden Jahrgängen an (Abb. 3). Die Erscheinung von in situ, iberwiegend wohl durch chemische Lösung entstandenen Facettenformen verdient besondere Aufmerksamkeit. 


\section{ANALYSE DER POSTMORTAL ENTSTANDENEN SCHALENABSCHLIFFE BEI CARDIUM EDULE}

Abgestorbene, doppelklappige Cardien mit den Frühformen der postmortalen Abschliffe zeigen, daß diese von verschiedenen Klappen-Ơffnungswinkeln abhängen sowie von der Verbindung durch das Schloß und dem Dehnungsvermögen des Ligamentes. Ihnen sind mit fortschreitender Bewegungsfreiheit zunehmend usurierte Flächen durch die sich gegenseitig abreibenden Klappen anzuschließen, Uberprägungen und Ausweitungen der primären Schlifformen der 1. bis 3. Modi. Da hier nur eindeutig postmortale Abschürfungen der Rippen berücksichtigt werden, fand nur ein geringer Anteil der zur Untersuchung vorliegenden Cardien Verwendung: An den über den Meeresboden driftenden Muscheln verwischen sich die Formen zunehmend durch Sandschliff und gehen ineinander über.

Im Versuch stellten sich erste schwache Abriebspuren am Periostrakum und damit Schalenaufhellungen bei Strömungsgeschwindigkeiten von 50 bis $70 \mathrm{~cm} / \mathrm{min}$ bereits nach $10 \mathrm{~min}$ ein!

\section{Morphologie und Funktion der sekundären Usuren}

Lebende Cardien öffnen ihre Klappen gewöhnlich in einem Winkel von 10 bis $15^{\circ}$. Eine Weile wird der gleiche Offnungswinkel noch nach dem Tode beibehalten, dann sperren sich die Klappen weiter auf. Für Exemplare mit eingetrockneten und geschrumpften Weichteilen beträgt der Winkel zunächst $10^{\circ}$, weitet sich jedoch nach Verlust des Weichkörpers auf 70 bis $120^{\circ}$ und schließlich bis über $180^{\circ}$. Unterschiedlich stark klaffende Schalen mit gedörrten Ligamenten finden sich oft auf den Wattflächen. Auch unter ständiger Wasserbedeckung spreizt das elastische Ligament die Klappen über das Maß hinaus, das zu Lebzeiten für aktive Offnungsbewegungen erforderlich wird (SCHÄFER 1962, p. 187). Die Cardien liegen dann auf einer Schale (postmortaler Flankenabschliff!) und sperren die andere Klappe nach oben ab. Bei mäßigen Wasserbewegungen hält das Ligament beide Klappen noch mehrere Monate nach dem Tode zusammen, die dann innen und außen von Balaniden besiedelt werden (Abb. 15). Je länger diese Cardien im Wasser liegen, desto nachgiebiger wird das Ligament, das schließlich auch seitwärts gerichtete Bewegungen der Klappen gegeneinander zuläßt. Bei den postmortalen Klappenöffnungen über $60^{\circ}$ reißt das Ligament ein, bei $70^{\circ}$ treten bereits kurze Risse, am Hinterende beginnend, auf. Bei etwa $120^{\circ}$ lösen sich die Schloßzähne voneinander. Der nunmehr allein wirksame, ligamentäre Zusammenhalt der voll seitenbeweglichen Klappen kann bis über $180^{\circ}$ hinaus erhalten bleiben (vgl. Abb. 22).

Die Frühformen der sekundären Usuren sind im wesentlichen den Offnungswinkeln 30 bis $120^{\circ}$ zuzuordnen und liegen daher auf den Muschelklappen über der Wirbelspitze. Gelegentlich treten sie isoliert auf oder werden von den primären Usuren durch einen Querstreifen mit Skulpturresten abgesetzt.

I. Allein durch Klapp-Bewegungen bei größeren Offnungswinkeln und den gegenseitigen Abrieb entstehen rückweisende, hochgelegene Schliffstreifen, die sich, bei einsetzender Seitenbeweglichkeit der Klappen, nach außen hin verbreitern. Selten ein- 
mal stehen die Schliffstreifen senkrecht zum Bauplan oder weisen nach vorne (Abb. 8 und 12).

II. Mit beginnender Torsion der Klappen gegeneinander bildet sich ein ausgebreiteter, halbkreisförmiger und weißgeschliffener Hof über der Wirbelspitze aus, in den zunehmend weitere Flächen einbezogen werden.

Klaffende Schalenklappen richten unter dem Einfluß von Strömungen den schwersten Teil des Gehäuses, die Wirbel- und Schloßregion, gegen die Grundströmung (Johnson 1957, Abb. 2; Schäfer 1962, Abb. 99) und sind damit Abschliffen ausgesetzt. Beim Trockenfallen der Watten schrumpt das Ligament und öffnet die Klappen: Im auflaufenden Wasser schwimmen diese Muscheln auf oder schleifen in der

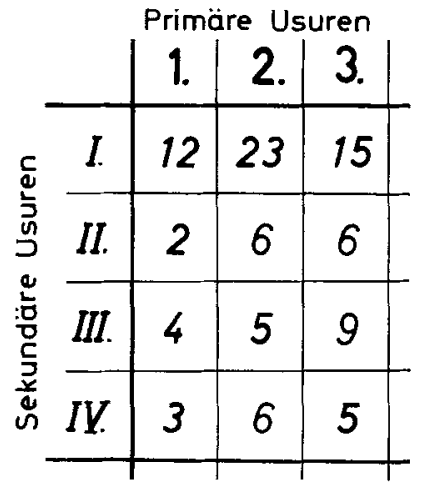

Abb. 22: Ubersicht über die durdischnittlichen \%/o-Anteile in den Jahresklassen 1961 bis 1965 , deren primäre Usurflächen (1.-3. Modus) durch postmortale, sekundäre Schlifformen (I.-IV.) überprägt werden. Der Schliffstreifen (I.) ist an den Exemplaren des Uthörner Außenwatts oft ausgebildet. Die übrigen sekundären Ábriebformen (II.-IV.) sind annähernd gleich häufig

Strömung über den Boden, Ursachen für dorsale und laterale Läsionen (KLÄHN 1932, pp. 376, 386). Gespreizte Klappen können in Grundströmungen jedoch auch über die kufenartigen, ventralen Schalenränder und den Dorsalbuckel rollen und werden auf diese Art weit über die Watten transportiert. Linke (1939, p. 277, Abb. 41) beobachtete, daß klaffende Schalen, gewölbt-oben, parallel zur Stromrichtung in regelmäßigen Reihen hintereinander liegen, die größeren weiter prielabwärts vertrieben und im Stromschatten der kleineren. Diese Gradierung könnte jedoch auch durch entgegengesetzt laufende Wasserströmungen entstehen.

Bei den aufgeführten Verdriftungen entstehen Schäden an der Skulptur des Dorsalbuckels beim Schleifen der Schalen über den Boden:

III. Die postmortale Querstriemung, teils durch Schliff, teils durch oberflächliches Absplittern der Schale, die in ihren Anfangsstadien unvollständig ist, von regelloseckigen Rändern begrenzt wird und Wachstumsunterbrechungen folgt.

IV. Der postmortale Buckel-Spiegel bildet sich beim Schwoien der gespreizten Klappen in Dorsalstellung auf dem Boden und wird anfangs in gleicher Weise unvollständig in die Schalen eingeschliffen (Abb. 14).

Keines der hier untersuchten Exemplare weist allseitig-flächenhafte Abnutzungen auf, die durch häufige Umlagerung und durch Sandschliff entstehen. 


\section{DISKUSSION}

Unsere Untersuchungen zielen in erster Linie auf mechanisch bedingte SchalenAbriebformen $a b$, bei denen sich zwei funktionsmorphologisch und ihrer Entstehung nach unterschiedliche Gruppen erkennen lassen. Sowohl zu Lebzeiten wie auch post mortem schleifen die ligamentär verbundenen Klappen aneinander: Dadurch entstehen primäre Usuren des 2. und 3. Modus (p. 184) sowie sekundäre Abnutzungen der I. und II. Form (p. 188), die in ihren Frühstadien eindeutig voneinander zu unterscheiden sind. Im Gegensatz dazu wird die Skulptur der Muschelschalen intra vitam und ebenso auch nach dem individuellen Tod auf dem Sedimentboden abgerieben: In charakteristischer Weise bei den primären Usuren des 4. und 5. Modus sowie sekundär in der III. und IV. Form.

Ergänzend wurden einige Schalen-Längs- und Querschliffe von C. edule und C. tuberculatum gefertigt. Beide Arten besitzen einen weitgehend übereinstimmenden Schalenbau, so daß das dickschalige und im Bild einprägsame C. tuberculatum zu bevorzugen ist (Abb. 16 bis 18 ), dessen primäre Usuren des 3. Modus markante Abriebformen zeigen. Bei C. edule sind viele der untersuchten Schäden oberflächlich; äußere Lamellen des Ostrakums werden zerstört. Abschliffe der Radialrippen reichen jedoch tiefer ins Schalengefüge hinein.

Läsionen der ontogenetisch ältesten Gehäusepartien durch bohrende Organismen, durch chemische Schalenkorrosion oder mechanisch abgeschliffene Dorsalbuckel stellen eine verbreitete Erscheinung bei den Lamellibranchiaten dar. Bei vielen Arten treten dabei sehr spezifische Veränderungen auf, die in enger Beziehung zum Schalenbauplan sowie zum Offnungsmechanismus stehen und damit ausgesprochen funktionsmorphologische Züge tragen. Berührungs- und Schleifkontakte an den Wirbelspitzen, die durch das Offnen und Schließen der Klappen eine zunehmend stark usurierte, glattgescheuerte Fläche ausbilden, sind bislang nicht allgemein, ihrer funktionsmorphologischen Bedeutung entsprechend, bekannt.

Anscheinend sind die Muscheln für die betrachteten Schliffschädigungen prädestiniert, deren Wirbelspitzen sich gegeneinander richten und über das Schloß hinausragen. C. edule, C. tuberculatum, C. echinatum, C. pancicostatum tragen ebenso wie auch Venerupis pullastra (Abb. 4 und 10) die verschiedenen Formen der primären und sekundären Usuren. Andere Bivalvia, wie lebende Venus gallina oder Cardita calyculata, sollten auf diese Gesetzmäßigkeit hin untersucht werden.

Zusätzlich begünstigen die mehrmals, in der Nordsee vier- bis achtmal, im Jahr gebildeten Zuwachslinien die Abschliffe der Dorsalbuckel oder im Gezeitenrhythmus erfolgende Vertikalbewegungen der Tiere im Substrat. Bei den C. edule aus der Tonnenlegerbucht können die primären Usuren durch chemische Schalenkorrosion oder Algen-Bohrungen verstärkt werden.

Am Berührungskontakt der Wirbelspitzen zu Lebzeiten eingeklemmte, grobe Quarzkörner, die in den primären Usuren des 3. Modus beider Klappen tief eingeschliffene Gruben hinterlassen, bieten auch postmortal einen guten Anhaltspunkt für den Bewegungsmechanismus der Muscheln, speziell für C. edule, deren Klappen sich allein öffnen oder schließen. Seitliche Verschiebungen der Klappen gegeneinander werden von lebenden Tieren nicht ausgeführt. 
Eigenartig sind die Querstriemungen, die symmetrisch auf beiden Buckeln hervortreten und die Inhomogenitäten im Schalenfeinbau der ältesten Schalenteile sichtbar machen. Mechanische, chemische und biologische Einflüsse führen an anderen Exemplaren zu Buckel-Spiegeln. Für ausgespülte, lebende C. edule ist eine aufrechte, stabile Dorsalstellung in der natürlichen Umgebung sowie im Seewasser-Aquarium in Ausnahmen zu beobachten.

Mechanisch kann diese Umkehrung der natürlichen Lebendstellung begründet werden. Uber die biologischen Ursachen und Zusammenhänge sind nur Vermutungen möglich: Vergleiche mit Dreissena polymorpha, Mytilus edulis oder den rezenten Limen bieten sich geradezu an (SEILACHER 1954).

Die Anwendung der Ergebnisse auf fossile Lamellibranchiaten erfordert gewisse Voraussetzungen: Untersuchung an intakten Muscheln, die ohne Umlagerung in situ eingebettet wurden und deren Schalen keine ausgeprägten diagenetischen Schäden zeigen, werden zu weitergehenden Rückschlüssen führen, zumal dann, wenn es sich um Arten mit empfindlichen Skulpturelementen handelt.

\section{ZUSAMMENFASSUNG}

1. An den Klappen des libero-hemisessilen Sandliegers Cardium edule L. entstehen zu Lebzeiten auffallende Abschliffe, die sich funktionsmorphologisch begründen und primäre Bau- und Härteunterschiede in den aragonitischen Schalen erkennen lassen. Die Abschliffe hängen mit typischen, häufig wiederholten Bewegungs- und Verhaltensweisen der Muschel im Sediment und an dessen Oberfläche zusammen, die am Meer und in einem Seewasserbecken beobachtet wurden.

2. Damit wird ein Modellfall zur Rekonstruktion solcher Lebensgewohnheiten vorgeführt, die an den überlieferten Hartteilen durch artspezifische Schädigungen nachzuweisen sind. Diese Methode kann auch bei fossilen, insbesondere bei bodenbezogen lebenden Mollusken angewandt werden.

3. Erkennbare Unschärfen gehen auf ökologische Faktoren, auf die unterschiedliche Empfindlichkeit der Skulptur und mögliche, postmortale Überprägungen zurück.

Für die stetige Förderung meiner Untersuchungen bin ich Herrn Prof. Dr. H. HöLder in Münster zu Dank verpflichtet. Besonderer Dank gilt der Deutschen Forschungsgemeinschaft, die die Unterwasser-Beobachtungen vor Helgoland ermöglichte, sowie Herrn Prof. Dr. O. KInNE, dem Leitenden Direktor der Biologischen Anstalt Helgoland. Für sachkundige Unterstützung an der Meeresstation Helgoland gilt mein Dank Dr. G. Uhlig, Herrn A. Holtmann und für weitere Beratungen sowie für die Beschaffung des Materials Dr. E. ZIEGELMEIER, Litoralstation List auf Sylt.

\section{ZITIERTE LITERATUR}

Anthony, R., 1905. Influence de la fixation pleurothétique sur la morphologie des mollusques acéphales dimyaires. Annls Sci. nat. (Zool.) Sér. 9: 1, 165-396.

Baggerman, B., 1954. Spatfall and transport of Cardium edule L. Archs néerl. Zool. 10, $315-342$. 
Bozkschoten, G. J., 1966. Shell borings of sessile epibiontic organisms as palaeo-ecological guides (with examples from the Dutch Coast). Palaeogeogr. Palaeoclim. Palaeoecol. 2, $333-379$.

Boettger, C. R., 1932. Die funktionelle Bedeutung der Rippung bei Landschneckengehäusen. Zool. Anz. 98, 209-213.

- 1933. Uber eine ungewöhnliche Standorts-Modifikation von Flußmuscheln (Unio crassus RETZ). Senckenbergiana 15, 334-337.

CARTER, R. M. 1968. On the biology and palaeontology of some predators of bivalved mollusca. Palaeogeogr. Palaeoclim. Palaeoecol. 4, 29-65.

Creer, G. A., 1960. The development of the lamellibranch Cardium edule L. Proc. zool. Soc. Lond. 135, 243-260.

Hecht, F. \& Matern, H., 1930. Zur Ökologie von Cardium edule L. Senckenbergiana 12, 361-368.

Hunter, W. R. \& Grant, D. C., 1962. Mechanics of the ligament in the bivalve Spisula solidissima in relation to mode of life. Biol. Bull. mar. biol. Lab., Woods Hole 122, 369-379.

Johnson, R. G., 1957. Experiments on the burial of shells. J. Geol. 65, 527-535.

Kessel, E., 1937. Schalenkorrosion bei lebenden Strandschnecken (Littorina littorea) und ihre Ursache. Zool. Anz. (Suppl. Bd) 10, 69-77.

- 1938. Über Erhaltungsfähigkeit mariner Molluskenschalen in Abhängigkeit von der Struktur. Arch. Molluskenk. 70, 248-254.

KLÄHN, H., 1932. Der quantitative Verlauf der Aufarbeitung von Sanden, Geröllen und Schalen in wässerigem Medium. Neues Jb. Miner. Geol. Paläont. Beil. Bd 67 B, 313-412.

Kreger, D., 1940. On the ecology of Cardium edule L. Arch. néerl. Zool. 4, 157-200.

Linke, O., 1939. Die Biota des Jadebusenwattes. Helgoländer wiss. Meeresunters. 1, 201-348.

Menard, H. W. \& Boucot, A. J., 1951. Experiments on the movement of shells by water. Am. J. Sci. 249, 131-151.

Mortensen, Th., 1921. Biologiske Studier over Sandstrandsfaunaen, saerlig ved de danske Kyster. Vidensk. Meddr dansk naturh. Foren. 74, 23-56 (1922).

Mosebach, R., 1952. Wässerige $\mathrm{H}_{2} \mathrm{~S}$-Lösungen und das Verschwinden tierischer Hartteile aus werdenden Sedimenten. Senckenbergiana 33, 13-22.

Orton, J. H., 1927. On the rate of growth of Cardium edule. J. mar. biol. Ass. U.K. 14, 239-279.

Pratje, O., 1929. Fazettieren von Molluskenschalen. Paläont. Z. 11, 151-169.

Remane, A., 1940. Einführung in die zoologische OKologie der Nord- und Ostsee. Tierwelt N.- H. Ostsee 1(1a), 1-238 (Lfg. 34).

SCHÄFER, W., 1950. Klaffmuschel-Spülsäume am Wattenstrand. Natur Volk 80, 173-176.

- 1962. Aktuo-Paläontologie nach Studien in der Nordsee. W. Kramer, Frankfurt a. M., $666 \mathrm{pp}$.

Schuster, O., 1951. Die Lebensgemeinschaften auf dem Südwatt der Nordseeinsel Mellum. Senckenbergiana 32, 49-65.

- 1952. Die Vareler Rinne im Jadebusen. Abb. senckenb. naturforsch. Ges. 486, 1-38.

SCHWARz, A., 1932. Der Lichteinfluß auf die Fortbewegung, die Einregelung und das Wachstum bei einigen niederen Tieren (Littorina, Cardium, Mytilus, Balanus, Teredo, Sabellaria). Senckenbergiana 14, 429-454.

SeILACHer, A., 1953. Der Brandungssand als Lebensraum in Vergangenheit und Vorzeit. Natur Volk 83, 263-272.

- 1954. Okologie der triassischen Muschel Lima lineata (Schlotн.) und ihrer Epöken. Neues Jb. Geol. Paläont. Mb. 1954, 163-183.

-.- 1960. Epizoans as a key to ammonoid ecology. J. Paleont. 34, 189-193.

Stephen, A. C., 1931. Notes on the biology of certain lamellibranchs on the Scottish Coast. J. mar. biol. Ass. U. K. 17, 277-300.

SWAN, E. F., 1952. The growth of the clam Mya arenaria as affected by the substratum. Ecology 33, 130-134.

Thamdrup, H. M., 1935. Beiträge zur Okologie der Wattenfauna auf experimenteller Grundlage. Meddr Kommn Danm. Fisk.-og Havunders. (Ser. Fisk.) 10 (2), 1-125. 
TRueman, E. R., 1954. Observations on the mechanism of the opening of the valves of a burrowing lamellibrand, Mya arenaria. J. exp. Biol. 31, 291-305.

VERWEY, J., 1954. On the ecology of distribution of cockle and mussel in the Dutch Waddensea, their rôle in sedimentation and the source of their food supply. Archs néerl. Zool. 10, 171-239.

VoGEl, K., 1959. Wachstumsunterbrechungen bei Lamellibranchiaten und Brachiopoden. Neues Jb. Geol. Paläont. Abb. 109, 109-129.

WohlenBerg, E., 1937. Die Wattenmeer-Lebensgemeinschaft im Königshafen von Sylt. Helgoländer wiss. Meeresunters. 1, 1-92.

Zregrlmeier, E., 1963. Das Makrobenthos im Ostteil der Deutschen Bucht nach qualitativen und quantitativen Bodengreifer-Untersuchungen in der Zeit von 1949-1960. Veröff. Inst. Meeresforsch. Bremerh. Sonderbd (1), 101-114.

- 1964. Einwirkungen des kalten Winters 1962/63 auf das Makrobenthos im Ostteil der Deutschen Bucht. Helgoländer wiss. Meeresunters. 10, 276-282. 